\title{
Exploring Barriers and Enablers to Dietitians Completing Body Composition Assessments as Part of Routine Clinical Care
}

Chloe Jobber

Department of Dietetics \& Foodservices, Mater Health, South Brisbane, Queensland

Shelley Wilkinson ( $\sim$ s.wilkinson@uq.edu.au )

School of Human Movement and Nutrition Sciences, The University of Queensland, Queensland

Elyssa Hughes

Department of Nutrition and Dietetics, Logan Hospital

Fiona Nave

Department of Dietetics \& Foodservices, Mater Health, South Brisbane, Queensland

Barbara van der Meij

Bond University Nutrition and Dietetics Research Group, Gold Coast

\section{Research Article}

Keywords: barriers, body composition assessment, enablers, malnutrition, sarcopenia

Posted Date: December 1st, 2020

DOI: https://doi.org/10.21203/rs.3.rs-113964/v1

License: (c) (1) This work is licensed under a Creative Commons Attribution 4.0 International License. Read Full License 


\section{Abstract}

Background:

Malnutrition, sarcopenia and cachexia are clinical wasting syndromes characterised by muscle loss. Systematic monitoring by body composition assessment $(\mathrm{BCA})$ is recommended for the diagnosis, treatment and monitoring of the syndrome(s). However, limited literature exists regarding integration of BCA into routine practice. This study investigated practices, competency, and attitudes of Australian dietitians regarding BCA, to inform a local implementation process.

\section{Methods:}

Applying the Action cycle in the Knowledge to Action framework, surveys were distributed to the 26 dietitians in our department. The survey assessed barriers and enablers to performing routine BCA in clinical care. Results were categorised using the Theoretical Domains Framework (TDF) and suitable interventions mapped using the Behaviour Change Wheel.

Results:

Twenty-two dietitians (84.6\%) completed the survey. Barriers to BCA were identified in all TDF domains. Enablers existed in domains of: Skills; Beliefs about consequences; Goals; Environmental context and resources; Social influences; Intentions; Optimism; Reinforcement.

\section{Conclusions:}

This study showed that hospital dietitians experience numerous individual, team, and organisational barriers to adopt BCAs in clinical practice. Information on barriers and enablers and implementation strategies have been suggested to overcome these barriers to assist BCA adoption into routine practice.

\section{Background}

Malnutrition, sarcopenia and cachexia are clinical wasting syndromes, prevalent in patients with acute or chronic diseases and frail elderly. ${ }^{1-3}$ Malnutrition occurs in $20-50 \%{ }^{1,4}$ of patients in acute care settings, sarcopenia in $15-70 \% 5,6$ and cachexia in $5-80 \% .{ }^{7-9}$ Malnutrition is defined as "a state resulting from lack of intake or uptake of nutrition that leads to altered body composition (decreased fat free mass) and body cell mass leading to diminished physical and mental function and impaired clinical outcome from disease". ${ }^{10}$ Malnutrition is associated with reduced treatment efficacy and increased healthcare costs. ${ }^{11,12}$ Disease-related malnutrition is characterised by inflammation and can be acute or chronic. Chronic disease-related malnutrition is also called 'cachexia' and is characterised by "inflammation and ongoing loss of weight and muscle mass". ${ }^{10}$ Sarcopenia is a condition which is often associated with malnutrition. It is defined as "loss of skeletal muscle mass and strength related to ageing and/or chronic disease", 13,14 and is associated with negative outcomes across health care settings including reduced survival, worse clinical outcomes and impaired quality of life in many clinical populations including oncology, surgical, hepatology, and older adults. ${ }^{15-17}$ As sarcopenia is prevalent amongst elderly and chronically ill, assessment and treatment has been encouraged by several leading expert groups. ${ }^{13,18}$ To be able to identify sarcopenia, assessment of muscle strength and muscle quantity or quality is required.

The three syndromes of malnutrition, cachexia, and sarcopenia are present in hospital populations and although they have been well defined in clinical practice the umbrella term 'malnutrition' is used for patients who show signs of inadequate food intake, weight loss, and muscle wasting. It is recommended to screen for malnutrition on admission to the hospital and regularly during hospital stay, and to treat malnutrition as early as possible. Malnutrition is typically 'managed' with a two-step process of screening and assessment. The initial step uses a malnutrition screening tool, such as the Malnutrition Screening Tool (MST), Nutritional Risk Screening (NRS) or Malnutrition Universal Screening Tool (MUST). ${ }^{10,19}$ Patients with screening scores above a certain number are classified as 'at risk of malnutrition' and subsequently referred to a dietitian. The second step is a dietitian assessment using a validated tool, such as the Subjective Global Assessment (SGA), or Patient-Generated Subjective Global Assessment (PG-SGA) or Mini-Nutritional Assessment (MNA). ${ }^{10,11}$ These assessment tools diagnose malnutrition by drawing on objective parameters such as weight and metabolic demand, as well as subjective parameters like weight history, nutrition impact symptoms, and physical examination of muscle mass and subcutaneous fat stores. ${ }^{20}$ Based on these measures, a patient is diagnosed as 'well nourished' (SGA/PG-SGA 'A') or 'no nutritional risk', 'moderately malnourished' (SGA/PG-SGA 'B') or 'at risk of malnutrition' or 'severely malnourished' (SGA/PG-SGA 'C') or 'malnourished'. The PG-SGA also produces a corresponding numerical score which provides a guideline to the level of nutrition intervention that is required. ${ }^{11}$ Nutritional assessment may include additional anthropometric assessments such as mid upper arm circumference, skin fold thickness and mid upper arm muscle circumference. ${ }^{21}$

Whilst parameters of nutrition assessment tools are easy to gather and rate highly regarding sensitivity, specificity and inter-rater reliability, they do not provide objective data on body composition such as muscle mass. ${ }^{22}$ In addition, there is a subset of patients who cannot be weighed and using an estimated weight leaves a margin for error in classifying malnutrition. A patient's perception of their weight loss can be incorrect, thus decreasing the efficacy of malnutrition assessment tools. ${ }^{23}$ Nutrition assessment tools also fail to recognise that patients can have a low level of lean tissue with any BMI category. ${ }^{24}$

Thus, measures of overall weight loss lack the sensitivity to detect the amount of lean mass an individual has and the potential loss of lean mass experienced. This introduces the potential to grossly underestimate the prevalence of hospital malnutrition if diagnosis is based on body weight and body weight changes alone. Other challenges that impact the correct identification of malnutrition are our ageing society and the global epidemic of overweight and obesity, resulting in a higher number of patients with sarcopenia, as well as overweight and obese patients with chronic or acute diseases. Malnutrition in these patients is harder to recognise using the aforementioned assessment tools, but is nonetheless associated with worse outcomes. ${ }^{24,25}$ 
As a result of these shortcomings there is a lack of consensus amongst the dietetic and medical community surrounding malnutrition assessment methodologies. ${ }^{17,26-31}$ With societal changes and improved technologies available, it is prudent to consider additional objective ways to obtain information on lean mass, in order to diagnose and monitor the effectiveness of the treatment of malnutrition. ${ }^{17,31,32}$

Routinely measuring lean mass provides an objective measure to diagnose and monitor malnutrition. This aligns with the international clinical nutrition community's recognition of the need for BCA as part of malnutrition assessment ${ }^{15,17,33}$ Indeed, the new Global Leadership Initiative on Malnutrition (GLIM) criteria recommends the measuring of body composition and identifying loss of lean mass as one of the top five criteria to assist in diagnosing malnutrition. ${ }^{27}$ Since the launch of the GLIM criteria, several initiatives have been taken to validate the criteria. These showed that the GLIM criteria have a fair agreement with the reference standard. ${ }^{34,35,36}$

Despite available evidence of the benefits of BCA, body composition is not routinely used by dietitians in clinical practice. This is reportedly due to incomplete knowledge and awareness, uncertainty of how and when to measure, poor availability of assessment tools and a lack of time. ${ }^{17,37}$ Given the role that lean mass plays in the clinical outcomes of certain illnesses, it is of critical importance that its assessment be added into the nutrition field. ${ }^{15,17}$

It is widely recognised that the dissemination of information alone does not change practice; ${ }^{38}$ thus drawing on an implementation science methodology facilitating this change and adoption process. ${ }^{39}$ This theory-driven approach guides the rigorous and systematic processes of evidence selection, adapting knowledge to the local context, understanding barriers and enablers to its use, selecting appropriate interventions to support its adoption, and monitoring and evaluating outcomes, as well as sustaining knowledge use, as outlined in the Knowledge-to-Action (KTA) framework. ${ }^{40}$ Within this framework additional theories, models, and frameworks can be applied to guide structured and systematic barrier identification and intervention selection, such as the Theoretical Domains Framework (TDF) and the Behaviour Change Wheel (BCW). ${ }^{41,42}$

The aim of this project was to develop a department-wide strategy to incorporate BCA by dietitians into routine clinical care using an Implementation Science approach. To inform this process we planned to investigate the current local practices, competency, and attitudes of our departmental clinical dietitians with regards to the utilisation of $\mathrm{BCA}$.

\section{Methods}

The study was declared as Exempt from Review - Not Research according to the Human Research Ethics Committee of Mater Research Institute - UQ Human Research Ethics Committee (Project ID: EXMT/MML/58778). All methods were carried out in accordance with relevant guidelines and regulations. All participants who completed surveys were informed their completion implied consent.

This implementation planning project occurred in an 800-bed tertiary hospital in Brisbane, Australia. The hospital provides services to both private and public inpatients and outpatients and includes a variety of patient populations. At the start of this project (May 2017), The Dietetics and Foodservices department consisted of 20.55 full time equivalents (FTE) with 26 dietitians.

To develop our BCA implementation strategy we followed the KTA framework which is an iterative approach that allows building (Knowledge Creation) and application of knowledge (Action Cycle). ${ }^{40}$ The Action Cycle was the focus of this work; with steps that can occur sequentially or concurrently and involve identification of the problem, assessing knowledge use determinants, evaluating the impact of knowledge use or outcomes, and ensuring sustainability. ${ }^{40}$ This project focused on the problem and barrier identification steps, as well as the intervention design step. In this project assessment and intervention selection required use of the $\mathrm{TDF}^{42}$ and $\mathrm{BCW} ;{ }^{41}$ the TDF as a system for categorising and defining barriers, and the BCW as a system for guiding decision-making around designing behaviour change interventions based on the identified barriers.

Below, we outline the survey process which allowed determination of a dietetic departmental practices, competency, and attitudes. A survey was developed to assess barriers and enablers to BCA use within the dietetic department (Appendix A). Questions were designed to map against domains of the TDF. ${ }^{42}$ Questions covered knowledge attitudes on, and confidence in BCA device use, frequency and predicted time taken to use the devices, views on how it would change dietetic practice, and which patient cohorts would benefit from BCA. All department dietitians were invited to complete the survey via an email link to an online survey portal (Survey Monkey, San Mateo, CA, USA) in May 2018. The survey was open for two weeks and two reminders were sent prior to the closing date.

The results were summarised as frequencies and percentages of answers for each question. All authors reviewed the summarised survey results and tabulated the barriers and enablers identified (Table 1). This process involved a content analysis using the TDF as the framework to categorise qualitative responses into themes; these responses were sorted into identified barriers and enablers, followed by documenting the source of the behaviour using the BCW (columns 4 and 5) and finally interventions were designed drawing from the implementation science literature. ${ }^{41,43}$ Findings were refined through group discussion resulting in consensus, with subsequent operationalisation and prioritisation of strategies.

\section{Results}

Twenty-two of 26 dietitians (84.6\%) completed the survey. As shown in Table 2, more than half of clinicians had previous training in BCA, mostly in skinfold thickness and mid upper arm circumference (MUAC). Few had training in bioelectrical impedance spectroscopy (BIS) devices. The majority of clinicians were aware that skinfold calliper, BIS, PG-SGA physical exam, hand grip dynamometer and tape measure devices were available for use in their department. More clinicians felt confident using PG-SGA physical exam and tape measures with fewer feeling confident using the BIS, MUAC and handgrip devices and 
techniques. As seen in Figure 1, the PG-SGA physical exam was the most common assessment reported to be performed, followed by the use of tape measures. The majority of clinicians reported that they never used skinfold measurement, BIS, MUAC or handgrip measures.

Dietitians' attitudes to use of BCA in routine practice were categorised into barriers and enablers (Table 1). Eight of the 22 staff (36.4\%) reported feeling that BCA measurements were more relevant for research or not appropriate in their area of work (TDF domain $\sim$ Social/professional role and identity). Over half of the staff (59.1\%) noted that BCA was not in their daily routine, they felt they were not able to accurately perform measurements (27.3\%) and that they wouldn't have time to complete them (18.2\%) (Belief about capabilities). Some respondents (13.6\%) felt measurements would not benefit their practice (Belief about consequences), and that there was a lack of procedures to perform measurements in departmental policies (31.8\%) (Environmental context and resources). Six dietitians (27.3\%) mentioned it would not fit their schedule, and seven dietitians found it 'too much of a hassle' to find reference values and report the results. Twelve dietitians (54.5\%) did not know how to schedule access to the devices, four (18.2\%) believed they did not have access to BCA devices and did not know how to get them to the ward and six (27.3\%) did not know where devices were kept.

However, positively, 16 dietitians (72.7\%) reported that they would like to learn more about incorporating BCA (Optimism) and 15 (68.2\%) would like to apply it to their practice. Two thirds $(n=14,74 \%)$ of dietitians reported they felt it would make their practice more interesting and under half $(n=10,45 \%)$ thought it would improve their practice. Twelve dietitians (55\%) felt more training would prompt them to perform assessments, and 14 (64\%) responded they would like the help of the clinical experts within the department (Reinforcement). Approximately half of the dietitians ( $n=10,45 \%)$ noted they would need to change their practice regarding assessment of nutritional status (Behavioural regulation).

Table 1 shows the mapping of the identified barriers and enablers (columns 2 and 3 ) to the TDF domains (column 1). Interventions and how these can be operationalised, drawing from the literature ${ }^{41,43}$ are in columns 5 and 6 . Barriers to use of BCA within our department were identified in all TDF domains of: Knowledge; Skills; Social/ professional role and identity; Beliefs about capabilities; Beliefs about consequences; Goals; Memory, attention and decision processes; Environmental context and resources; Social influences; Intentions; Emotion; Optimism; Reinforcement and Behavioural regulation. Enablers included: Skills; Beliefs about consequences; Goals; Environmental context and resources; Social influences; Intentions; Optimism; Reinforcement.

Through the detailed mapping process, these are summarised in and operationalised in column 6 of Table 1. They can broadly be grouped as: 1. Professional development strategy, 2. Body composition assessment clinical champion project, and 3. Departmental integration process.

\section{Discussion}

This study aimed to understand the attitudes, beliefs, and practices of clinicians in a tertiary hospital dietetics department regarding patients' BCA practices to inform a process of integrating these practices into routine clinical care. Most dietitians rarely used BCA with their patients in a systematic way. Barriers and enablers existed in many of the same TDF domains. Many dietitians felt unsure of their skills, when and how to systematically use these BCA techniques, and some questioned their benefit for particular clinical areas (e.g. neonatal care) and/or outside of research projects. However, many dietitians were optimistic about the potential this process would provide to enabling evidence-based practice and noted it would add to the strength of assessments, recommendations, and ability to detect malnutrition and other wasting syndromes, and to clinically relevant improvements within the delivery of medical nutrition therapy.

To our knowledge, this is the first study to investigate barriers and enablers to systematic adoption of BCA techniques into routine dietetic clinical practice. While many papers have promoted the use of BCA to detect malnutrition, ${ }^{10,15,17,27,30,31}$ and specific studies described the application of these techniques in clinical areas (e.g. elderly; ${ }^{32}$ liver failure; ${ }^{44-46}$ oncology; ${ }^{47,48}$ renal disease ${ }^{49}$ and respiratory disease ${ }^{26}$ ) none have applied this across a hospital dietetics department.

To our knowledge, only one study, by Reijnierse et al (2017), documented barriers to BCA application in practice. ${ }^{37}$ These were explored before and after a Dutch health professional training program on detection and management of sarcopenia. ${ }^{37}$ Barriers included lack of availability of equipment, lack of knowledge, time constraints, and lack of collaboration with/awareness of other health professionals. ${ }^{37}$ When Reijnierse's study was repeated in a similar sized cohort of Australian and New Zealand health professionals $(n=250)$, as previously found, a lack of diagnostic tools was the main reason for not diagnosing sarcopenia. ${ }^{50}$ Lack of sarcopenia awareness and lack of motivation among health-care professionals were also common barriers. ${ }^{50}$ In addition to most of these, our study identified additional barriers relating to clinicians' beliefs about the applicability of the techniques, personal ability to undertake the assessments and confidence in their abilities to incorporate these into their daily practice. Our more extensive suite of barriers may have resulted from a more profession-specific/department-wide assessment rather than training attendees of varied professions. ${ }^{37,50}$

Moreover, applicability issues also relate to BCA validity issues when used with acutely or chronically ill patients. American Society for Parenteral and Enteral Nutrition's (ASPEN) recent systematic review showed minimal studies that have provided data on BCA in clinical populations. Out of BIA, DXA and ultrasound, DXA and CT scanning were recommended as 'gold standard', but the authors indicated that more research is required on the validity of BCA in specific patient populations. ${ }^{30}$

Acknowledging the need for addressing all "bottlenecks" (barriers) in each phase of the implementation to ensure diagnosis and management of sarcopenia in daily practices, Reijnierse et al (2017) highlight the need to draw on the implementation science literature in delivering effective interventions. ${ }^{37}$ They highlight that this requires many factors such as acquisition of diagnostic measurement devices, reorganisation of care, collaboration between healthcare professionals, perceived needs and benefits of innovation and organizational factors. ${ }^{37}$ Accordingly, we have adopted an implementation science approach to ensure we systematically select interventions that align with identified barriers and enhance existing enablers. ${ }^{40-42}$ 
Following the operationalisation of the evidence-informed strategies to overcome the identified barriers and enablers, our team will progress the overarching interventions of upskilling (professional development strategy), modelling and reducing fear of change (clinical champion project) and embedding as usual practice (departmental integration) the use of BCA. ${ }^{41,43}$ The details that will be incorporated into these strategies are in the final column of Table 2 . We will repeat our departmental survey in mid-2020 to re-assess adoption of, (perceived) competency in, and attitudes of clinical dietitians towards the utilisation of BCA devices within our department.

A study strength included the use of implementation science methodology and frameworks (KTA, TDF, BCW) ${ }^{40-42}$ to map and inform our strategy. Many solutions may appear 'common sense' but the systematic assessment and rigour provided by the process provides confidence in the findings and interventions. The survey revealed numerous barriers and enablers to the adoption of BCA in routine clinical care. A greater understanding and/or a wider selection of barriers may have been identified through more qualitative approaches (e.g. focus groups, interviews). However, the methodological approaches were pragmatically chosen to be administered and analysed within routine practice without additional funding. The barriers and enablers identified may reflect specific local departmental issues and may not be generalisable to all sites. However, it is likely that many of these issues are common to other Australian and international sites, as highlighted by Reijnierse ${ }^{37}$ and Yeung ${ }^{50}$ and colleagues. Study limitations include potential reporting bias or answers reflecting social desirability despite being an anonymous due to the small team size plus lack of data on time burden for dietitians of performing measurements, booking devices, and carrying devices to clinics or wards. We also lack data on objective clinical practice change, resultant clinical outcomes, and cost-effectiveness. Another limitation is the potential impact of knowledge and practice loss with staff turnover; however this was attempted to be circumvented with handover and orientation processes.

\section{Conclusions}

In summary, malnutrition is associated with poorer clinical outcomes in hospitalised patients. BCA devices can be a useful addition to routine clinical care to detect muscle loss that can otherwise be undetected in current malnutrition screening and assessment processes. However, we identified numerous health professional, team, and organisational barriers to the systematic adoption of these processes. Through a process of barrier analysis and intervention mapping within an implementation science framework we have designed three-pronged strategy of dietitian upskilling, embedding and evaluating, and managementendorsement and support to facilitate adoption of practices that will support evidence-based care for these patients. We aim to successfully implement BCA into routine dietetic practise in our hospital department. Future research is required to investigate the validity for each disease, health care setting and age category, as well as to show the impact on clinical practice, clinical outcomes and health economics.

\section{List Of Abbreviations}

BCA Body Composition Assessment

BCW Behaviour Change Wheel

BIS Bioelectrical Impedance Spectroscopy

DXA Dual Energy X-ray Absorptiometry

FTE Full Time Equivalents

GLIM Global Leadership Initiative on Malnutrition

KTA Knowledge to Action

MUAC Mid Upper Arm Circumference

MUST Malnutrition Universal Screening Tool

MST Malnutrition Screening Tool

PG-SGA Patient-Generated Subjective Global Assessment

SGA Subjective Global Assessment

TDF Theoretical Domains Framework

\section{Declarations}

\section{Ethics approval and consent to participate}

The study was declared as Exempt from Review - Not Research according to the Human Research Ethics Committee of Mater Research Institute - UQ Human Research Ethics Committee (Project ID: EXMT/MML/58778). All methods were carried out in accordance with relevant guidelines and regulations. All participants who completed surveys were informed their completion implied consent.

\section{Consent for publication}


Not applicable.

\section{Availability of data and materials}

The datasets used and/or analysed during the current study are available from the corresponding author on reasonable request.

\section{Competing interests}

The authors declare that they have no competing interests.

\section{Funding}

Dr Shelley Wilkinson was supported by a Queensland Government Department of Health- Health Research Fellowship.

\section{Authors' contributions}

All authors have participated sufficiently in the article to take public responsibility for the content. Chloe Jobber was responsible for collecting and analysing data, interpreting results, writing the manuscript. A/Prof Shelley Wilkinson (corresponding author) and Dr Barbara van der Meij were responsible for study design, project coordination, data interpretation and manuscript preparation. Elyssa Hughes was responsible for collecting and analysing data, and reviewing the manuscript. Fiona Nave was responsible for collecting data, coordinating the project, and reviewing the manuscript.

\section{Acknowledgements}

Queensland Health - Health Research Fellowship funding (2015-2020).

\section{References}

1. Barker LA, Gout BS, Crowe TC. Hospital malnutrition: prevalence, identification and impact on patients and the healthcare system. Int J Environ Res Public Health. 2011;8(2):514-27.

2. Lim SL, Ong KC, Chan YH, Loke WC, Ferguson M, Daniels L. Malnutrition and its impact on cost of hospitalization, length of stay, readmission and 3-year mortality. Clin Nutr. 2012;31(3):345-50.

3. Correia M, Perman MI, Waitzberg DL. Hospital malnutrition in Latin America: A systematic review. Clin Nutr. 2017;36(4):958-67.

4. Agarwal E, Ferguson M, Banks M, Bauer J, Capra S, Isenring E.Nutritional status and dietary intake of acute care patients: results from the Nutrition Care Day Survey 2010. Clin Nutr. 2012;31(1):41-7.

5. Ryan AM, Power DG, Daly L, Cushen SJ, Ní Bhuachalla Ë, Prado CM. Cancer-associated malnutrition, cachexia and sarcopenia: the skeleton in the hospital closet 40 years later. Proc Nutr Soc. 2016 May;75(2):199-211.

6. Peterson SJ, Braunschweig CA. Prevalence of Sarcopenia and Associated Outcomes in the Clinical Setting. Nutr Clin Pract. 2016 Feb;31(1):40-8.

7. Gingrich A, Volkert D, Kiesswetter E, Thomanek M, Bach S, Sieber CC, et al. Prevalence and overlap of sarcopenia, frailty, cachexia and malnutrition in older medical inpatients. BMC Geriatr. 2019 Apr 27;19(1):120.

8. De Waele E, Demol J, Caccialanza R, Cotogni P, Spapen H, Malbrain ML, et al.Unidentified cachexia patients in the oncologic setting:Cachexia UFOs do exist. Nutrition. 2019 Jul-Aug;63-64:200-204.

9. von Haehling S, Anker MS, Anker SD. Prevalence and clinical impact of cachexia in chronic illness in Europe, USA, and Japan: facts and numbers update J Cachexia Sarcopenia Muscle. 2016 Dec;7(5):507-509.

10. Cederholm T, Barazzoni R, Austin P, Ballmer P, Biolo G, Bischoff SC, et al. ESPEN guidelines on definitions and terminology of clinical nutrition. Clin Nutr. 2017 Feb;36(1):49-64.

11. Kellett J, Kyle G, Itsiopoulos C, Naunton M, Luff N. Malnutrition: The Importance of Identification, Documentation, and Coding in the Acute Care Setting. J Nutr Metab. 2016;2016:9026098.

12. Cederholm T, Jensen GL. To create a consensus on malnutrition diagnostic criteria: A report from the Global Leadership Initiative on Malnutrition (GLIM) meeting at the ESPEN Congress 2016. Clin Nutr. 2017;36(1):7-10.

13. Cruz-Jentoft AJ, Bahat G, Bauer J, Boirie Y, Bruyere O, Cederholm T, et al. Sarcopenia: revised European consensus on definition and diagnosis. Age and Ageing. 2019;48(1):16-31.

14. Bauer J, Morley JE, Schols A, Ferrucci L, Cruz-Jentoft AJ, Dent E, et al. Sarcopenia: A Time for Action. An SCWD Position Paper. J Cachexia Sarcopenia Muscle. 2019;10(5):956-61.

15. Earthman CP. Body Composition Tools for Assessment of Adult Malnutrition at the Bedside: A Tutorial on Research Considerations and Clinical Applications. JPEN J Parenter Enteral Nutr. 2015;39(7):787-822.

16. Martin L, Gioulbasanis I, Senesse P, Baracos VE. Cancer-Associated Malnutrition and CT-Defined Sarcopenia and Myosteatosis Are Endemic in Overweight and Obese Patients. JPEN J Parenter Enteral Nutr. 2020;44(2):227-238.

17. Deutz NEP, Ashurst I, Ballesteros MD, Bear DE, Cruz-Jentoft AJ, Genton L, et al. The Underappreciated Role of Low Muscle Mass in the Management of Malnutrition. J Am Med Dir Assoc. 2019;20(1):22-7. 
18. Beaudart C, Rolland Y, Cruz-Jentoft AJ, Bauer JM, Sieber C, Cooper C, et al. Assessment of Muscle Function and Physical Performance in Daily Clinical Practice: A position paper endorsed by the European Society for Clinical and Economic Aspects of Osteoporosis, Osteoarthritis and Musculoskeletal Diseases (ESCEO). Calcif Tissue Int. 2019 Jul;105(1):1-14.

19. Ferguson M, Capra S, Bauer J, Banks M. Development of a valid and reliable malnutrition screening tool for adult acute hospital patients. Nutr. 1999;15(6):458-64.

20. Bauer J, Capra S, Ferguson M. Use of the scored Patient-Generated Subjective Global Assessment (PG-SGA) as a nutrition assessment tool in patients with cancer. EJCN. 2002;56(8):779-85. Epub 2002/07/18.

21. BAPEN. (2020), Nutritional Assessment. https://www.bapen.org.uk/nutrition-support/assessment-and-planning/nutritional-assessment?showall=1 (Accessed 10 November 2020).

22. Kyle UG, Pirlich M, Lochs H, Schuetz T, Pichard C. Contribution of body composition to nutritional assessment at hospital admission in 995 patients: a controlled population study. Br J Nutr. 2001;86(6):725-31.

23. Benítez Brito N, Suárez Llanos JP, Fuentes Ferrer M, Oliva García JG, Delgado Brito I, Pereyra-García Castro F, et al. Relationship between Mid-Upper Arm Circumference and Body Mass Index in Inpatients. PloS one. 2016;11(8):e0160480.

24. Martin L, Birdsell L, Macdonald N, Reiman T, Clandinin MT, McCargar LJ, et al. Cancer cachexia in the age of obesity: skeletal muscle depletion is a powerful prognostic factor, independent of body mass index. J Clin Oncol. 2013;31(12):1539-47.

25. World Health Organization (2018). Overweight and obesity. https://www.who.int/news-room/fact-sheets/detail/obesity-and-overweight. (Accessed 3 December 2019).

26. Walter-Kroker A, Kroker A, Mattiucci-Guehlke M, Glaab T.A practical guide to bioelectrical impedance analysis using the example of chronic obstructive pulmonary disease. Nutr J. 2011;10:35.

27. Cederholm T, Jensen GL, Correia M, Gonzalez MC, Fukushima R, Higashiguchi T, et al. GLIM criteria for the diagnosis of malnutrition - A consensus report from the global clinical nutrition community. Clin Nutr. 2019;38(1):1-9.

28. Kyle U, Morabia A, Slosman D, Mensi N, Unger P, Pichard C. Contribution of body composition to nutritional assessment at hospital admission in 995 patients: a controlled population study. Br J Nutr. 2001;86(6):725-31.

29. Kyle UG, Pirlich M, Lochs H, Schuetz T, Pichard C.Increased length of hospital stay in underweight and overweight patients at hospital admission: a controlled population study. Clin Nutr. 2005;24(1):133-42.

30. Sheean P, Gonzalez MC, Prado CM, McKeever L, Hall AM, Braunschweig CA. American Society for Parenteral and Enteral Nutrition Clinical Guidelines: The Validity of Body Composition Assessment in Clinical Populations. JPEN J Parenter Enteral Nutr. 2019.

31. Thibault R, Pichard C. The evaluation of body composition: a useful tool for clinical practice. Ann Nutr Metab. 2012;60(1):6-16.

32. Dent E, Hoogendijk EO, Visvanathan R, Wright ORL.Malnutrition Screening and Assessment in Hospitalised Older People: a Review. J Nutr Health Aging. 2019;23(5):431-41.

33. Mareschal J, Achamrah N, Norman K, Genton L. Clinical Value of Muscle Mass Assessment in Clinical Conditions Associated with Malnutrition. J Clin Med. 2019;8(7):1040.

34. Allard JP, Keller H, Gramlich L, Jeejeebhoy KN, Laporte M, Duerksen DR. GLIM criteria has fair sensitivity and specificity for diagnosing malnutrition when using SGA as comparator. Clin Nutr. 2020 Sep;39(9):2771-2777.

35. Clark AB, Reijnierse EM, Lim WK, Maier AB. Prevalence of malnutrition comparing the GLIM criteria, ESPEN definition and MST malnutition risk in geriatric rehabilitation patients: RESORT. Clin Nutr. 2020;39(11):3504-3511.

36. Zhang X, Tang M, Zhang Q, Zhang KP, Guo ZQ, Xu HX, et al. The GLIM criteria as an effective tool for nutrition assessment and survival prediction in older adult cancer patients. Clin Nutr. 2020;10:S0261-5614(20)30405-2.

37. Reijnierse E, de van der Schueren M, Trappenburg M, Dvoes M, Meskers C, Maier A. Lack of knowledge and availability of diagnostic equipment could hinder the diagnosis of sarcopenia and its management PLoS One. 2017;12:e0185837.

38. Grol R, Wensing M. What drives change? Barriers to and incentives for achieving evidence-based practice. MJA. 2004;180(6):s57-60.

39. Eccles M, Grimshaw J, Walker A, Johnston M, Pitts N. Changing the behaviour of healthcare professionals: The use of theory in promoting the uptake of research findings. J Clin Epidemiol. 2005;58(2):113-6.

40. Straus S, Tetroe J, Graham I. Knowledge translation in health care. Moving from evidence to practice. Oxford: Wiley-Blackwell/BMJ Books; 2009.

41. Michie S, van Stralen M, West R. The behaviour change wheel: A new method for characterising and designing behaviour change interventions. Implement Sci. 2011;6:42

42. Francis J, O'Connor D, Curran J. Theories of behaviour change synthesised into a set of theoretical groupings: introducing a thematic series on the theoretical domains framework. Implement Sci. 2012;7:35.

43. Colquhoun H, Leeman J, Michie S, Lokker C, Bragge P, Hempel S, et al. Towards a common terminology: a simplified framework of interventions to promote and integrate evidence into health practices, systems, and policies. Implement Sci. 2014;9:51.

44. Montano-Loza AJ. Clinical relevance of sarcopenia in patients with cirrhosis. World J Gastroenterol. 2014;20(25):8061-71.

45. Hsu CS, Kao JH. Sarcopenia and chronic liver diseases. Expert Rev Gastroenterol Hepatol. 2018;12(12):1229-44.

46. Cichoz-Lach H, Michalak A. A Comprehensive Review of Bioelectrical Impedance Analysis and Other Methods in the Assessment of Nutritional Status in Patients with Liver Cirrhosis. Gastroenterol Res Pract. 2017;2017:6765856.

47. Crawford J. What are the criteria for response to cachexia treatment? Ann Palliat Med. 2019;8(1):43-9..

Page $7 / 16$ 
48. Carneiro IP, Mazurak VC, Prado CM. Clinical Implications of Sarcopenic Obesity in Cancer. Curr Oncol Rep. 2016;18(10):62. Epub 2016/08/20.

49. Carrero JJ, Johansen KL, Lindholm B, Stenvinkel P, Cuppari L, Avesani CM. Screening for muscle wasting and dysfunction in patients with chronic kidney disease. Kidney Int. 2016;90(1):53-66. Epub 2016/05/10.

50. Yeung S, Reijnierse E, Trappenburg M, Meskers C, Maier A. Current knowledge and practice of Australian and New Zealand health-care professionals in sarcopenia diagnosis and treatment: Time to move forward! Australas J Ageing. 2020; 39(2):e185-e193.

\section{Tables}

Table 1. Intervention mapping and operationalising after sorting of barriers and enablers to TDF domains from the dietitian's surveyed. 


\begin{tabular}{|c|c|c|c|c|}
\hline $\begin{array}{l}\text { TDF domain (and } \\
\text { definition) (26) }\end{array}$ & $\begin{array}{l}\text { Survey identified } \\
\text { Barriers }\end{array}$ & $\begin{array}{l}\text { Survey } \\
\text { identified } \\
\text { Enablers }\end{array}$ & $\begin{array}{l}\text { BCW Intervention } \\
\text { components (25) }\end{array}$ & $\begin{array}{l}\text { Definition of intervention with } \\
\text { examples of } \\
\text { Behaviour Change Techniques } \\
\text { (27) }\end{array}$ \\
\hline
\end{tabular}

\section{Knowledge -}

An awareness of the existence of

something
Unsure of clinical areas BCA would benefit

$75 \%(n=12)$ unsure who to use on;

$69 \%(n=11)$ unsure when to do;

$69 \%(n=11)$ unsure what to do;

$62.5 \%(n=10)$ unsure how to interpret;

\section{Psychological capability}

Education

Increasing knowledge or understanding:

E.g

Feedback on the behaviour/ outcome(s) of the behaviour

Self-monitoring of behaviour/ of outcome of behaviour

Prompts/cue

Information about social and environmental consequences

Information about others' approval

Imparting skills

Training

Enablement

Reducing barriers to increase capability or opportunity (beyond education, training and environmental restructuring)

E.g

Social support

Reduce negative emotions

Conserve mental resources

Self-monitoring of behaviour and outcome of behaviour

Graded tasks

Adding objects to the environment

Restructuring the social environment

Focus on past success

Verbal persuasion about capability

Self-reward

Goal setting (behaviour, outcome)

Commitment

Action planning

Review behaviour and outcome goal(s) 
Discrepancy between current behaviour and goal

Problem solving

Pros and cons

Monitoring of emotional consequences

Anticipated regret

\section{Skills-}

An ability or proficiency acquired through

practice
Don't know how to use $37.5 \%(n=6)$;

Lack of confidence (and enabler) $25 \%(n=4)$; Don't have time to perform $25 \%(n=4)$

More than $75 \%$ never use: skinfold $100 \%$, BIS $90 \%$, handgrip

95\%, MUAC 75\% ( $n=20$ responders)

$75 \%(n=12)$ unsure who to use on;

$69 \%(n=11)$ unsure when to do;

$69 \%(n=11)$ unsure what to do; $62.5 \%(n=10)$ unsure how to interpret;
Training and awareness in a variety of areas

- Had any training in BCA: $55 \%(n=22)$, mostly in arm circumference (75\%), skinfolds $(75 \%), \mathrm{BIS}(42 \%)$ and BIS scale $(42 \%)$. For DXA, only $8 \%$ had any training

- Had any training in BCA: $55 \%(n=22)$, mostly in MUAC (75\%), skinfolds (75\%), BIS (42\%) and BIS scale (42\%). For DXA, only $8 \%$ had any training

- The majority of the team (at least $75 \%$ ) is aware that skinfold callipers, BIS device, PG-SGA, handgrip, and tape measures are available. A minority is aware of the existence of a BIS scale $(n=20)$

- $\quad$ Confident to use skinfold (5\%), BIS (30\%), MUAC $70 \%$, PG-SGA $85 \%$, handgrip $50 \%$, tape measure $75 \%(n=20)$

\section{Reflective}

motivation (Cognitive/ interpersonal skills)

Education

Persuasion
Increasing knowledge or understanding

E.g. as above
Using communication to induce positive or negative feelings or stimulate action

E.g. Feedback on the behaviour/ on the outcome(s) of the behaviour,

Focus on past success,

Verbal persuasion about capability,

Persuasive source

Identity associated with changed behaviour

Identification of self as role model,

Information about social and environmental consequences,

Information about health consequences,

Salience of consequences,

Information

about others' approva,I

Social comparison

Creating expectation of reward

E.g.

Feedback on behaviour or

on the outcome(s)

of behaviour,

Self-monitoring of behaviour or outcome of behaviour,

Monitoring of (outcome of) behaviour by others without evidence of feedback

Situation-specify reward, 
Reduce reward frequency,

Incentivisation

Reward alternate behaviour

Remove punishment,

Social reward,

Self-reward,

Behavioural contract

Commitment

Discrepancy between current behaviour and goal

Creating expectation of punishment or cost

As above

Coercion

Physical capability

(physical skills)

Training

Page $11 / 16$ 


\begin{tabular}{|c|c|c|c|c|}
\hline \multirow{5}{*}{$\begin{array}{l}\text { Social/ professional } \\
\text { role and identity- } \\
\text { A coherent set of } \\
\text { behaviours and } \\
\text { displayed personal } \\
\text { qualities of an } \\
\text { individual in a social or } \\
\text { work setting }\end{array}$} & $N=6$ & Reflective motivation & As 'Skills' & 1 \\
\hline & \multirow{2}{*}{$\begin{array}{l}\text { I think these measures } \\
\text { are more appropriate } \\
\text { for research } 67 \%(n=4)\end{array}$} & Education & & \\
\hline & & Persuasion & & \\
\hline & \multirow{2}{*}{$\begin{array}{l}\text { I do not think these } \\
\text { measurements are } \\
\text { appropriate for my area } \\
\text { of work } 67 \%(n=4)\end{array}$} & Incentivisation & & \\
\hline & & Coercion & & \\
\hline \multirow{2}{*}{$\begin{array}{l}\text { Beliefs about } \\
\text { capabilities- }\end{array}$} & $37.5 \%(n=6)$ & Reflective motivation & \multirow[t]{5}{*}{ As 'Skills' } & t \\
\hline & \multirow{3}{*}{$\begin{array}{l}\text { I don't think I could } \\
\text { perform these measures } \\
\text { accurately }\end{array}$} & Education & & \\
\hline \multirow{3}{*}{$\begin{array}{l}\text { Acceptance of the } \\
\text { truth, reality, or validity } \\
\text { about an ability, talent, } \\
\text { or facility that a person } \\
\text { can put to constructive } \\
\text { use }\end{array}$} & & Persuasion & & t \\
\hline & & Incentivisation & & a \\
\hline & $\begin{array}{l}\text { I do not have time to } \\
\text { perform these } \\
\text { measurements } 25 \% \\
(n=4)\end{array}$ & Coercion & & \\
\hline
\end{tabular}

\section{Beliefs about consequences-}

Acceptance of the truth, reality, or validity about outcomes of a behaviour in a given situation

I think these measures
are more appropriate for
research $67 \%(n=4)$
Don't think these
measurements would
benefit my
practice/tell me
anything
new/useful 30\% ( $=3)$
I do not expect these
measurements to
change my practice 11\%
(n=2)

\section{$N=19$ overall Leverage for nasogastric tubes $26 \%(n=5)$}

Leverage for pre-surgical provision of enteral/parenteral nutrition $26 \%$ $(n=5)$

Assist in persuading patients to increase intake/supplements $63 \%$ $(\mathrm{n}=12)$

Assist in motivation (i.e. to continue on weight loss journey) $79 \%(n=15)$

Ability to more accurately assess energy requirements $89 \%(n=17)$

Ability to provide objective measures/ evaluations of dietetic interventions $84 \%$ $(n=16)$

Assist in identifying malnutrition $58 \%(n=11)$

Would make practice more interesting $74 \%(n=14)$

Would improve my practice $53 \%(n=10)$

With training and time, they (BCAs) could become routine $(n=1)$

\section{Reflective motivation}

As 'Skills'

Education

Persuasion

Incentivisation

Coercion 
Memory, attention and decision processes-

The ability to retain information, focus selectively on aspects of the environment and choose between two or more alternatives
Not in my daily routine $81 \%(n=13)$

Too much time to do $46 \%(n=6)$

I forget to do $31 \%(n=4)$

Hassle to find reference ranges $54 \%(n=7)$

Difficulties -

practicalities $(n=1)$
Applicable in some patient groups $(n=1)$

Great that we will have suppor to routinize $(n=1)$

\section{Psychological capability}

As 'Knowledge'

Education

Training

Enablement
If you can get access to the peapod for routine assessments that would be great $10 \%(n=1)$

We do not have procedures or forms to report these

measurements $70 \%$

$(n=7)$

development of skills

and abilities,

independence, social

competence, and adaptive behaviour
$\mathrm{N}=15$ overall

I don't have access to

the devices I need to

perform body

composition

assessment $27 \%(n=4)$

I don't know where these devices are

kept $40 \%(n=6)$

I don't know how to book these

devices $80 \%(n=12)$

I know where these devices are kept but I don't know how to get them to the

ward $27 \%(n=4)$

\section{Physical opportunity}

Restrictions

Using rules to reduce the opportunity to engage in the target behaviour (or to increase the target behaviour by reducing the opportunity to engage in competing behaviours)

Changing the physical or social context

Environmental restructuring I feel this would add value to
Dietitians and patient care in
relevant populations

\section{Social opportunity}

Restrictions

Persuasion
My peers do not perform these measurements, so why should

I? $31 \%(n=4)$

I think they are burdensome to patients $8 \%(\mathrm{n}=1)$

\section{Intentions-}

A conscious decision to perform a behaviour or a resolve to act in a certain way
$\mathrm{N}=18$ overall

I never think of doing these measurements when I see or evaluate a patient $39 \%(n=7)$

Not in my daily routine $81 \%(n=13)$
I would like to know more about what technology we have available and where it would be applicable.

I would certainly consider integrating into practice if and where appropriate.

I would like to add these measurements to my daily routine
Reflective motivation

Education

Persuasion

Incentivisation

Coercion
As above 
A complex reaction

pattern, involving

experiential,

behavioural, and

physiological elements,

by which the individual

attempts to deal with a

personally significant

matter or event
Don't want to break

device 13/\% $(n=2)$

Feel stressed about the

time

required $43 \%(n=6)$
Persuasion

Incentivisation

Coercion

Environmental restructuring

Modelling

Enablement

\section{Optimism-}

The confidence that things will happen for the best or that desired goals will be attained

\section{Reinforcement-}

Increasing the probability of a response by arranging

a dependent

relationship, or

contingency, between

the response and a

given stimulus

\section{$\mathrm{N}=19$}

Unsure if it'll be burdensome to patients $8 \%(n=1)$

Unsure how perceptive the patients will be $7 \%$ $(n=1)$

\section{$N=18$}

Nothing that prompts me $22 \%(n=4)$

I am ready - bring it on $(n=1)$
Would improve my practice
$(n=10)$
Will make practice more
interesting ( $n=14)$
May increase patients'
motivation to see me to get
results ( $n=1)$
More training would prompt me
(n=12)
Body comp team makes this
possible $(n=14)$

Integrate into WARs

\section{Education \\ Persuasion \\ Incentivisation \\ Coercion}

Reflective motivation

Automatic motivation As above

Persuasion

IncentivisationCoercion

Environmental restructuring

Modelling

Enablement

As 'Skills'
Psychological capability As 'Knowledge'

Training

Enablement
Happy to practice if

patient care $(n=1)$
Happy with the way assess nutritional status $22 \%(n=4)$
Would need to change practice $39 \%(n=7)$
I would need to change my practice regarding assessing nutritional status $56 \%(n=10)$
$N=18$

Education

'Knowledge'

objectively observed or

measured actions

\section{Behavioura
regulation-}

Anything aimed at 


\begin{tabular}{|c|c|c|}
\hline$\%(n)$ & & Dietitians \\
\hline Response rate & & $84.6(22)$ \\
\hline \multirow[t]{2}{*}{ Previous training in $\mathrm{BCA}$ use } & Yes & $54.5(12)$ \\
\hline & No & $45.5(10)$ \\
\hline \multirow[t]{4}{*}{ Previous training in BCA devices } & Skinfold callipers & $40.9(9)$ \\
\hline & Mid-upper arm circumference (MUAC) & $40.9(9)$ \\
\hline & $\begin{array}{l}\text { Bioelectrical impedance spectroscopy } \\
\text { (BIS) }\end{array}$ & $22.7(5)$ \\
\hline & Dual x-ray absorptiometry (DXA) & $4.5(1)$ \\
\hline \multirow[t]{6}{*}{ Knowledge of available of devices and procedures in department } & PG-SGA physical exam & $86.4(19)$ \\
\hline & BIS & $81.8(18)$ \\
\hline & Tape measures & $77.3(17)$ \\
\hline & Skinfold callipers & $68.2(15)$ \\
\hline & Handgrip & $68.2(15)$ \\
\hline & Bioelectrical impedance scale & $40.9(9)$ \\
\hline \multirow{6}{*}{$\begin{array}{l}\text { Rating of confidence in using BCA devices or undertaking procedures (extremely / reasonably } \\
\text { confident) }\end{array}$} & PG-SGA physical exam & $77.3(17)$ \\
\hline & Tape measures & $68.2(15)$ \\
\hline & MUAC & $63.6(14)$ \\
\hline & Handgrip dynamometer & $45.5(10)$ \\
\hline & BIS & $27.3(6)$ \\
\hline & Skinfold callipers & $4.5(1)$ \\
\hline
\end{tabular}

BCA - body composition assessment; BIS - Bioelectrical impedance spectroscopy; DXA - dual x-ray absorptiometry; MUAC- mid upper arm circumference; PGSGA - patient generated subjective global assessment; REE - resting energy expenditure

\section{Figures}




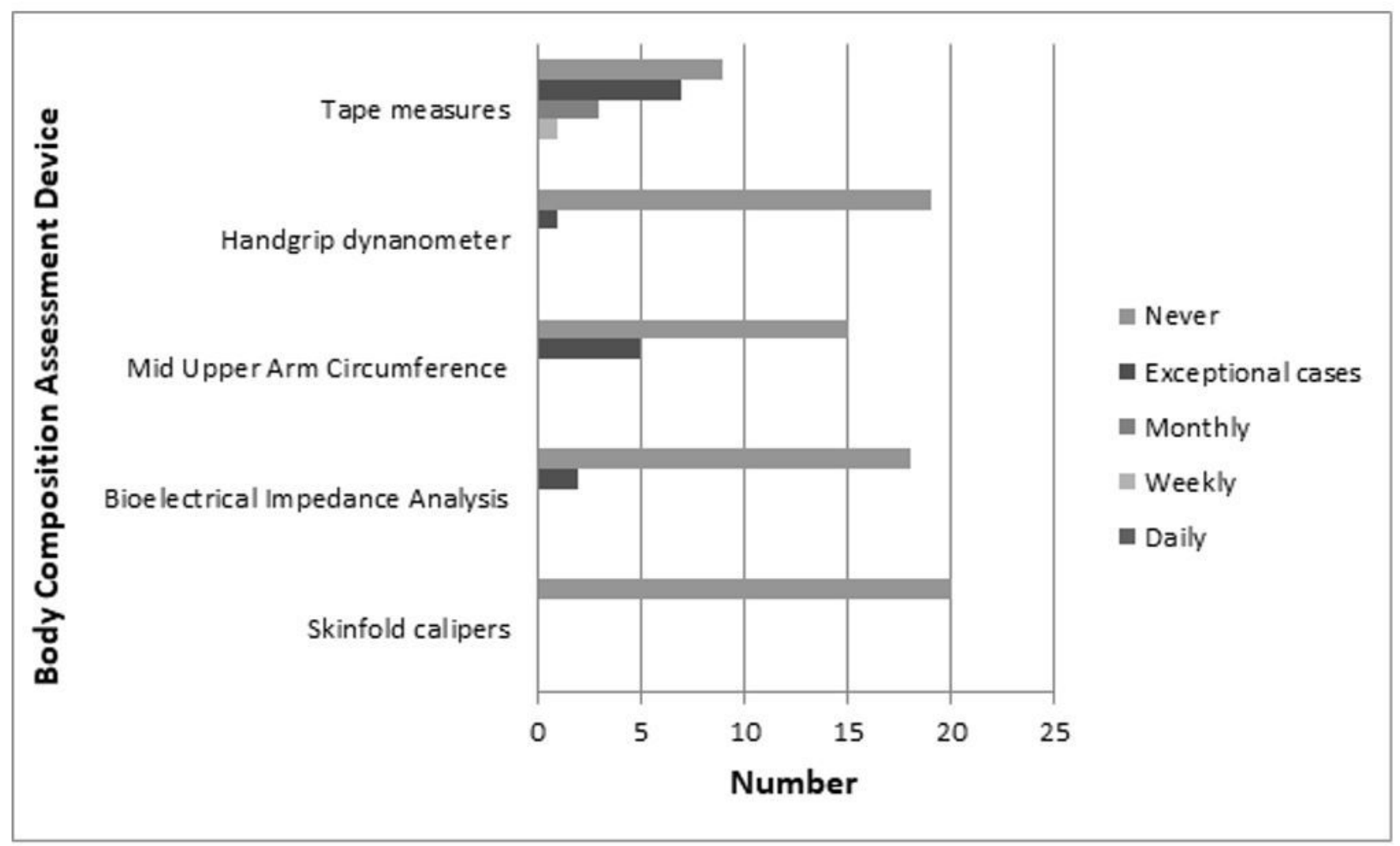

Figure 1

Frequency of reported device use by dietitians in routine clinical care.

\section{Supplementary Files}

This is a list of supplementary files associated with this preprint. Click to download.

- Additionalfile1.docx 